\title{
Sox9 and programming of liver and pancreatic progenitors
}

\author{
Yoshiya Kawaguchi \\ Department of Clinical Application, Center for iPS cell Research and Application (CiRA), Kyoto University, Kyoto, Japan.
}

\begin{abstract}
Recent advances in developmental biology have greatly expanded our understanding of progenitor cell programming and the fundamental roles that Sox9 plays in liver and pancreas organogenesis. In the last 2 years, several studies have dissected the behavior of the Sox $9^{+}$duct cells in adult organs, but conflicting results have left unanswered the long-standing question of whether physiologically functioning progenitors exist in adult liver and pancreas. On the other hand, increasing evidence suggests that duct cells function as progenitors in the tissue restoration process after injury, during which embryonic programs are sometimes reactivated. This article discusses the role of Sox9 in programming liver and pancreatic progenitors as well as controversies in the field.
\end{abstract}

\section{Introduction}

Mechanisms of organogenesis and adult organ maintenance have attracted the interest of not only basic scientists but also clinicians, because such research deepens our understanding of disease pathogenesis and provides a basis for exploring new therapies. Indeed, the proposed concept of cancer stem/initiating cells, which is essentially analogous to stem cells in organogenesis, has been experimentally validated for some tumors, and researchers hope to develop therapeutics that specifically target these cells (1-5). Notably, many similarities exist between the cellular processes of tissue regeneration and tumorigenesis, such as aggressive cell proliferation, migration, and epithelial-mesenchymal transition, in which embryonic gene sets are sometimes reactivated (6-10). This notion illustrates the importance of developmental biology research and, in particular, the characterization of organ-specific progenitors.

Sex-determining region Y-box (SRY-box) containing gene 9 (Sox9), a member of the SRY-related, high-mobility group box (Sox) transcription factors, is mutated in campomelic dysplasia, a disorder characterized by skeletal malformations, XY sex reversal, and neonatal lethality (11). Sox9 is expressed during embryogenesis in several tissues and organs including chondrocytes, testes, heart, lung, pancreas, bile duct, hair follicles, retina, and the central nervous system (12-21). Sox9 functions, in general, to maintain cells in an undifferentiated state during embryonic development. In the last few years, the role of Sox9 in the organogenesis and maintenance of adult liver and pancreas has been vigorously investigated. However, conflicting results exist on the behavior of adult Sox $9^{+}$cells (22-24). In this article, rather than simply rehashing the latest studies, I attempt to dissect the role of Sox9 in the programming of the liver and pancreas progenitors and to provide clinical aspects of diseases related to Sox 9 .

\section{Cre-based genetic lineage tracing uncovers cell behavior in vivo}

To understand the behavior of specific cells in vivo, either during organogenesis or in the adult organ, reliable analytical methods must be applied. An important advance in lineage-tracing

Conflict of interest: The author has declared that no conflict of interest exists. Citation for this article: J Clin Invest. 2013;123(5):1881-1886. doi:10.1172/JCI66022. experiments was the application of the Cre/loxP system (ref. 25 and Figure 1). In this method, mice carry 2 transgenic gene cassettes: (a) a Cre recombinase gene expression cassette that is driven from a cell type-specific promoter (by conventional transgenesis, bacterial artificial chromosome [BAC] transgenesis, or knock-in to the endogenous locus of a cell type-specific marker gene), and (b) the floxed reporter allele whose expression is activated by Cre-mediated recombination (such as ROSA26r, in which a loxP-STOP-loxP- $\beta$-galactosidase gene cassette is knocked in to the ubiquitously expressed ROSA26 locus) (26). To mark cells in a temporal manner, Cre can be fused with the ligand-binding domain of the estrogen receptor (CreER or its variants), rendering its activity tamoxifen inducible. The addition of tamoxifen drives the CreER fusion protein into the nucleus. Recombination disrupts the STOP cassette within the reporter allele and thereby permanently marks cells by reporter expression. Because recombination occurs within the genomic DNA, this mark is inherited by all descendants of the labeled cell regardless of its subsequent cell fate(s).

\section{Sox9 in embryonic pancreas and liver}

Developmentally, the pancreas and liver originate from the foregut endoderm. In the pancreas, Sox 9 is expressed in all epithelial cells at early developmental stages; its expression is confined to the duct cells as development proceeds. Differentiated acinar and endocrine cells do not express Sox9 $(22,27)$. The fate of embryonic Sox $9^{+}$cells was independently analyzed using different Sox9-CreER mouse lines. Lineage-tracing experiments using either BAC Sox9-CreER transgenic (23) or Sox9-IRES-CreER knock-in mice (22) showed that all types of pancreatic epithelial cells including endocrine, acinar, and duct cells were labeled, suggesting a common origin from Sox9-expressing progenitors. This notion is supported by another pseudo-short-term cell-tracking experiment that took advantage of the half-life of GFP in Sox9-EGFP transgenic mice (27).

What is the role of Sox9 in pancreatogenesis? Pathological analyses on autopsy samples from 3 humans with campomelic dysplasia showed impaired pancreas formation described as "less densely packed epithelial cells within the mesenchymal stroma and less clearly formed islets" (28). Campomelic dysplasia is an autosomal-dominant disease caused by SOX9 haploinsufficiency, suggesting a dosage requirement for Sox9 in pancreatic development; 


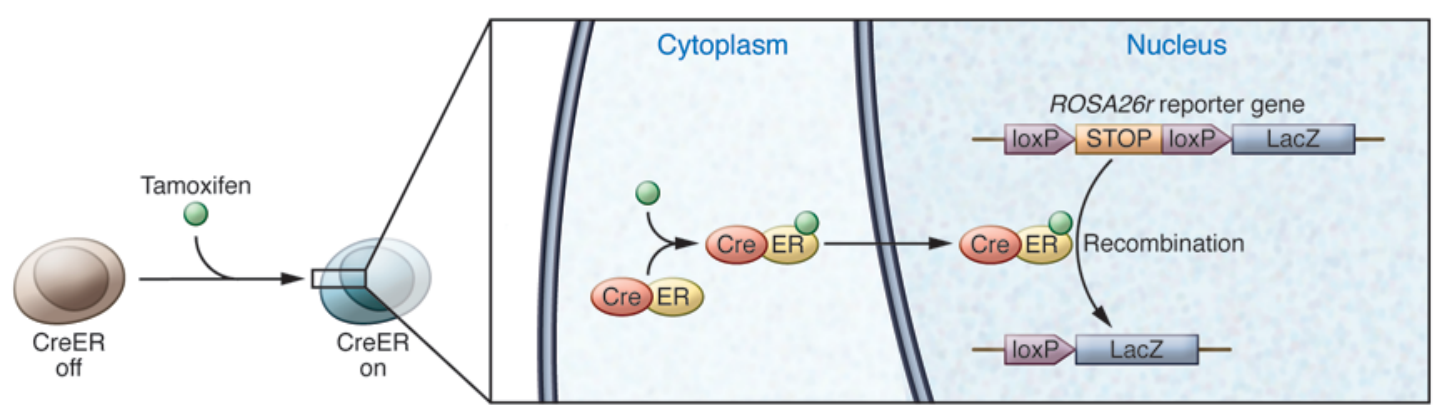

\section{Figure 1}

Tamoxifen-inducible Cre-mediated lineage tracing. Cre recombinase is fused with the ligand-binding domain of the estrogen receptor. Tamoxifen administration enables the CreER fusion protein to enter the nucleus then excise the STOP cassette of ROSA26r, resulting in permanent labeling of the progeny. Such strategies have been instrumental to understanding the role of Sox9 in pancreatic and liver progenitor cells.

this notion is consistent with observations that Sox9 heterozygous mutant mice die perinatally $(11,16,29,30)$. When Sox 9 gene dosage was experimentally reduced to $50 \%$ in murine pancreatic progenitors, the number of neurogenin 3-expressing (Ngn3-expressing) endocrine progenitors was reduced, resulting in less endocrine cell formation, whereas exocrine mass was not impaired (27). These findings indicate that the pancreatic endocrine lineage is more sensitive than the exocrine lineage to Sox 9 dosage, in accordance with the endocrine-dominant impairment in cases of humans with campomelic dysplasia (28). On the other hand, pancreas-specific Sox9 depletion in mice resulted in severe pancreas hypoplasia, suggesting that Sox 9 functions in the expansion of the pool of multipotent progenitor cells by accelerating their proliferation and survival and by keeping them in an undifferentiated state in early pancreatic development (16).

How is Sox9 regulated during pancreatogenesis? Recent reports showed that the involvement of FGF and Notch signaling pathways are conserved between mouse and zebrafish (refs. 31-35 and Figure 2). During early pancreatic development, FGF receptor $2 \mathrm{~b}$ (Fgfr2b) expressed in the Sox $9^{+}$epithelium transduces a mesenchymally derived Fgf10 signal. Expression of Fgfr2b is regulated by Sox9. Moreover, Fgf10 is required to maintain the expression of Fgfr2b and Sox9 in pancreatic progenitors. This Fgf10/Fgfr2b/ Sox9 feed-forward loop in the early pancreatic niche is pivotal not only for the expansion of the pancreatic precursors, but also for the maintenance of organ identity (33). Inactivation of either Fgf10 or Sox 9 in pancreatic progenitors results in the emergence of $\alpha$-fetoprotein-positive cells in the pancreatic rudiment, suggesting a conversion in organ identity from pancreas to liver (33).

\section{Figure 2}

Maintenance of pancreatic fate and gene regulation in the programming of pancreatic endocrine progenitors. A Fgf10/Fgfr2b/Sox9 feed-forward loop functions in the maintenance of pancreatic fate and expansion of early pancreatic precursors. Sox9 promotes the expression of Fgfr2b, which transduces signaling from Fgf10. Moreover, Fgf10 signaling promotes the expression of Fgfr2b and Sox9 in pancreatic progenitors. In the endocrine differentiation, a parallel regulatory circuitry exists, involving Notch, Hes1, Sox9, and Ngn3 (31-33).
In pancreatic endocrine differentiation, there exists a complicated but well-organized molecular circuitry involving Sox9, Notch, Hes1, and Ngn3 (ref. 32 and Figure 2). Notch signaling activates Sox9 expression within the primitive epithelium. Sox9, in turn, is required for the induction of $\mathrm{Ngn} 3$, an indispensable gene for endocrine differentiation. At the same time, Ngn3 is negatively regulated by the Notch effector Hes1 $(32,36,37)$. Thus, Ngn3 is regulated by the balance of Sox 9 and Hes1. At high Notch activity, Hes1 activity predominates over Sox9, resulting in Ngn3 downregulation. To complete endocrine differentiation, Sox9 expression is subsequently silenced. In this process, cell-autonomous repression of Sox 9 by $\mathrm{Ngn} 3$ and evasion from Notch activity seems to be crucial. At the same time, Sox9 regulates Hes1, and the number of $\mathrm{Hes}^{+}$cells is reduced by Sox9 depletion (16). These results suggest the existence of a mutual regulatory system between Sox9 and Notch/Hes1/Ngn3 signals, in which dosage or strength of the signals controls the proliferation and differentiation of the pancreatic endocrine precursors (32). In contrast, regulation of Sox 9 in exocrine pancreatic development has been poorly investigated. One hypothesis is that a similar mechanism operates in exocrine precursors, with pancreas-specific transcription factor 1a (Ptf1a) substituting for Ngn3. Ptf1a initially functions as the pancreatic-determinant gene during the fate separation of the primitive gut epithelia into the duodenum, pancreas, and bile duct lineages $(38,39)$. In addition, Ptfla is indispensable for acinar cell differentiation $(38,40)$. Dosage control of Ptfla is important for both functions; Ptf1a reduction causes the fate conversion of pancreatic precursors into the duodenal and bile duct cells and decelerates proliferation and differentiation in the exocrine lineage (39). In addition, autoregulation of Ptf1a to maintain Ptf1a dosage plays a part in the 


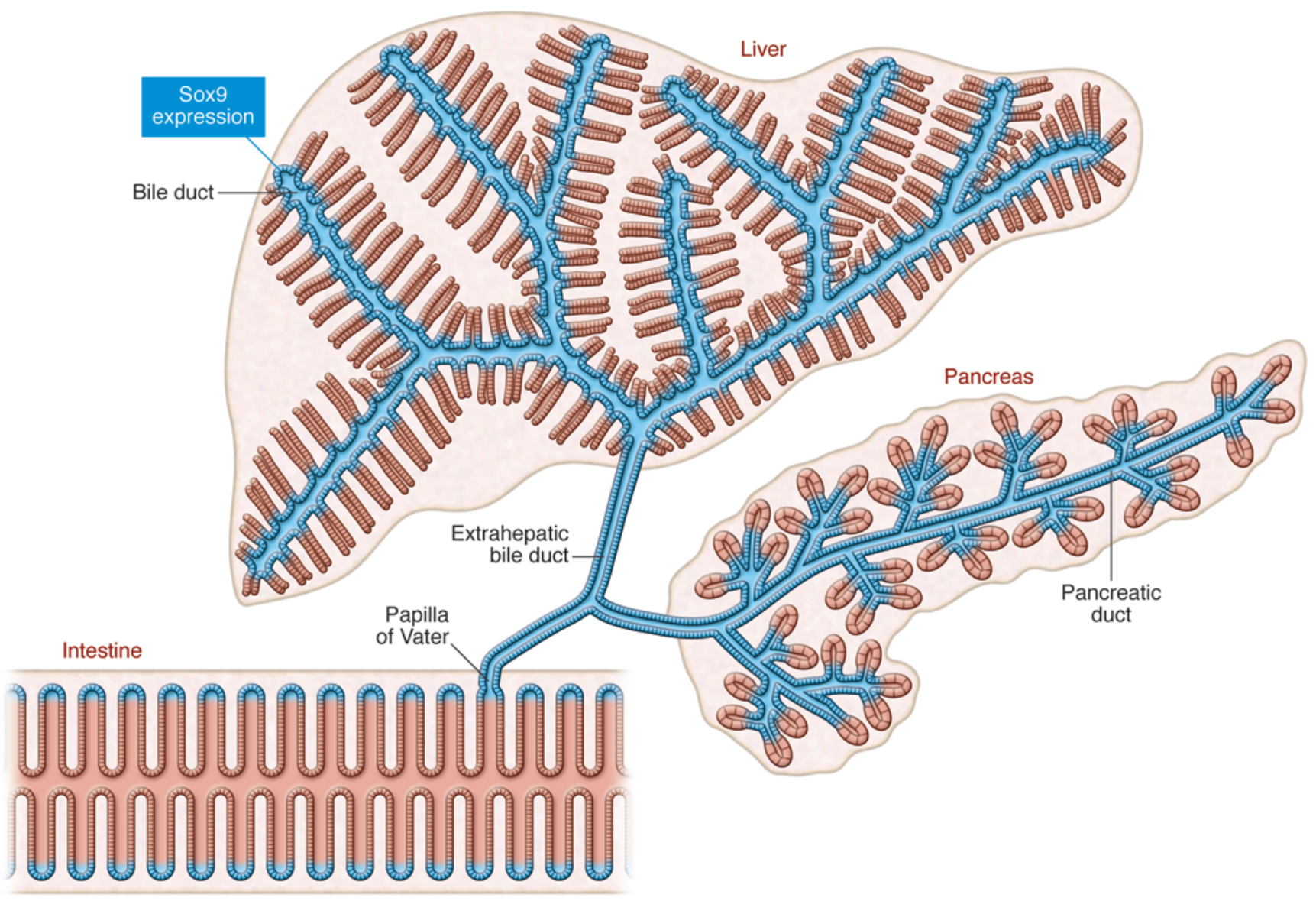

Figure 3

The continuous Sox9-expressing domain in adults. Sox9 expression is detected throughout the pancreatic ducts, intra- and extrahepatic ducts, and in the intestinal crypt connected through the papilla of Vater, forming a contiguous Sox $9^{+}$zone.

final maturation of the acinar cells $(41,42)$. Like Ngn3, Ptf1a is also negatively regulated by Hes1 (43). These lines of evidence support the hypothesis that Sox9 and a Notch/Hes1/Ptfla axis control exocrine development, but future experiments are needed for confirmation.

In liver development, hepatoblasts and hepatocytes do not express Sox9, but Sox9 is detected in cholangiocytes $(17,22)$. Notably, lineage tracing using Sox9-IRES-Cre knock-in mice provided evidence that embryonic Sox $9^{+}$cholangiocytes can differentiate into hepatocytes, although only a subset of hepatocytes was labeled, predominantly in the hilar region at P1 (22). Similar results were also obtained using BAC Sox9-CreER transgenic mice (44). Construction of the intrahepatic biliary tree originates in the hilum and expands toward the periphery. Morphologically, tubular formation of the ducts begins with the formation of an asymmetrical primitive ductal structure in which Sox $9^{+}$cholangiocytes are located on the portal side and Sox9- hepatoblasts are on the parenchymal side (17). After a maturation step involving TGF- $\beta$ signaling, the biliary tube is entirely composed of Sox $9^{+}$cholangiocytes. Embryonic liver-specific inactivation of Sox9 in mice resulted in a delay in duct maturation. Thus, Sox 9 determines the timing of bile duct morphogenesis (17). In addition, Notch definitively functions in the formation of bile duct structure and regulates Sox 9 as seen in the etiology of Alagille syndrome, an autosomal-dominant genetic disorder caused by mutations in the Notch pathway $(17,45-48)$. Notch regulation of Sox9 in the liver may be functionally analogous to its role in the pancreas, but Notch targets in the liver that correspond to Ngn3 and Ptf1a in pancreas formation have yet to be identified.

\section{Do physiologically functioning stem/progenitor cells exist in adult liver and pancreas?}

To maintain the functional homeostasis of an adult organ, which is composed of several cell types, either in the physiological state or during the regenerative process after injury, an orchestrated mechanism must ensure the correct supply or elimination of each cell type and the maintenance of tissue architecture. In the skin and gut, organ-specific stem cells located in the bulge of the outer root sheath of hair follicles and those in the intestinal crypt, respectively, continuously supply new cells, while the homeostatic elimination of old cells occurs in the skin surface and at the top of the intestinal villi, respectively (49, 50). Along with cell replacement in a streaming manner, tissue architecture is maintained. How such homeostasis is achieved in the case of the adult liver and pancreas remains an important question for the long-term efficacy of cell transplantation therapies such as proposed hepatocyte transplantation in pediatric patients (51).

In the 1980s, Zajicek et al. proposed a "streaming hypothesis" from their observations that adult hepatic cells marked by triti- 
ated thymidine incorporation migrated from the periportal region to the central vein region under physiological condition in rats (52-58). However, in the 1990s a heated debate on the streaming hypothesis erupted based on Bralet's experiments showing that rat hepatic cells marked by the retrovirus-mediated $\beta$-galactosidase gene expression did not stream (59). More recently, another report provided support for the streaming liver hypothesis (60). Fellous et al. showed that patches of cytochrome c oxidase-deficient hepatocytes that abut the portal tracts expanded toward the hepatic vein in human liver (60). Because cytochrome c oxidase is encoded by mitochondrial DNA, they used mitochondrial DNA as a marker of clonal expansion. They demonstrated that cytochrome c oxidase-deficient patches were clonal, suggesting an origin from long-lived cells such as stem cells located in the periportal region (60). Lineage-tracing experiments using Sox9-IRES-CreER knock-in mice and BAC Sox9-CreER transgenic mice demonstrated that adult Sox $9^{+}$bile duct cells at the periportal region physiologically supply new hepatocytes $(22,24)$. In Sox9-IRES-CreER knock-in mice, the newly formed hepatocytes appear to migrate very slowly from the periportal space to the central vein, as observed in the experiments with high-dose tamoxifen treatment (5 tamoxifen injections of $4 \mathrm{mg} / 20 \mathrm{~g}$ body weight), supporting the original "streaming hypothesis" (22). However, hepatocyte differentiation from the Sox $9^{+}$cells was limited to the periportal region in BAC Sox9-CreER transgenic mice (24). One criticism that arose against the tamoxifen-inducible Cre-based experiments is that tamoxifen injection causes hepatocyte toxicity and may result in ectopic Sox9 expression in hepatocytes. Indeed, a single injection of $10 \mathrm{mg} / \mathrm{kg}$ body weight of tamoxifen causes ectopic Sox 9 expression in hepatocytes within 18 hours (44); however, this cannot explain the results of the previously mentioned Sox9 lineage-tracing experiments, since ectopic Sox 9 expression is not restricted to periportal hepatocytes.

More recently, 2 tamoxifen-independent tracing experiments were reported $(61,62)$. Malato et al. used AAV-mediated hepatocyte labeling that argued against physiologically functioning progenitors in ducts (61). In this report, virus-mediated induction of Cre recombinase under the hepatocyte-specific transthyretin (Ttr) promoter in ROSA26-YFP reporter mice successfully labeled all the hepatocytes without any leaky labeling of Sox $9^{+}$duct cells. Following a 12-week chase, the liver did not contain yellow fluorescent protein-negative (YFP-) hepatocytes, indicating that hepatocyte mass is maintained solely through hepatocyte proliferation and that Sox $9^{+}$duct cells do not participate in maintaining adult organ homeostasis by differentiating into hepatocytes. On the contrary, Iverson et al. performed unique experiments that provided evidence of new hepatocyte supply in adults (62). Using Albumin-Cre;ROSA ${ }^{m T-m G}$ mice that convert from tdTomato expression to EGFP expression via Cre-mediated recombination, they revealed that a steady-state level of $0.076 \%$ of all hepatocytes had differentiated within the previous 4 days from albumin-naive precursors (62).

There has been a long-standing debate about whether physiologically acting stem/progenitor cells exist in the ductal structure of the adult pancreas. Studies have shown that embryonic Sox9 ${ }^{+}$duct cells can differentiate into pancreatic acinar and endocrine cells $(22,23)$. Because Sox9 expression persists throughout the epithelium of the adult pancreatic ductal tree, including centroacinar cells (Figure 3 ) (22), much interest has focused on the differential ability of adult Sox $9^{+}$cells. Lineage-tracing experiments using BAC Sox9-CreER transgenic mice demonstrated that Sox $9^{+}$duct cells lose their differentiation ability within a few days after birth. Thus, adult Sox $9^{+}$duct cells do not function as stem/progenitor cells in BAC Sox9-CreER transgenic mice (23). This finding is in accordance with another lineage-tracing experiment targeted to adult pancreatic cells; adult Hnf $1 \beta^{+}$ductal cells do not differentiate into acinar/endocrine cells (63). Moreover, pulse and chase experiments using RIP-CreER (64) or elastase-CreER (65) supported the notion that adult pancreatic $\beta$ cells and acinar cells are maintained by the self-duplication of preexisting cells rather than by differentiation from progenitors (66). Taken together, most of these results refute the existence of stem/precursor cells in the adult pancreatic duct. On the contrary, lineage tracing using Sox9-IRES-CreER knock-in mice showed essentially identical results to BAC Sox9-CreER transgenic mice during embryonic and neonatal stages, but differences between these two Sox9-CreER lines were seen in adult pancreas. In contrast to BAC Sox9-CreER-labeled cells, adult pancreatic Sox $9^{+}$ducts continuously supply new acinar cells in Sox9-IRES-CreER knock-in mice (22).

In examining these conflicting results from different Sox9-CreER lines, 2 potential pitfalls of the lineage-tracing experiments should be considered. First, differences in the initially marked cell population would naturally cause different results. For example, there exist Hes $1^{+}$ and Hes $1^{-}$cells in pancreatic ducts. Thus, lineage-tracing results of adult Hes $1^{+}$cells do not directly negate the existence of precursors in ducts. A detailed comparison of CreER expression in the different Sox9-CreER lines would be required to assess such variation. A more probable explanation is that the cell behavior is unexpectedly altered by the insertion of the transgenic cassette into the Sox9 locus. In Sox9-IRES-CreER knock-in mice, the dosage of Sox9 expression might be altered even though the introduced CreER cassette contains an IRES sequence. Preliminary data from our lab showed that Sox9 expression levels in the pancreas and liver were not altered in newborn pups but were reduced in adult Sox9-IRES-CreER knock-in mice compared with wild-type mice (Shinichi Hosokawa and Masashi Horiguchi, personal communication). This observation suggests that the 3 ' non-coding region of the Sox9 locus that is altered by the IRES-CreER cassette insertion (22) is pivotal for Sox9 regulation in adults but not in embryonic and newborn stages. Though not yet experimentally confirmed, we suspect that the dosage of Sox 9 is not altered in BAC Sox9-CreER transgenic mice. Thus, not only during development, but also in the adult organs, Sox9 expression levels appear to be crucial for controlling the cell status of the duct cells. Additional experiments, such as reducing the Sox9 dosage in adult pancreatic duct cells combined with lineage tracing using BAC Sox9-CreER, are of interest.

Kopinke et al. reported that adult Hes $1^{+}$cells occasionally observed in the pancreatic ducts and centroacinar cells do not differentiate into acinar cells in a physiological state, but that inactivation of Rbpj (also known as recombining binding protein suppressor of hairless) in Hes $1^{+}$cells causes rapid differentiation into acinar cells $(67,68)$. Thus, Notch functions to preserve the pancreatic duct cell identity in the ductal tree. Considering that Ptf1a is negatively regulated by Hes 1 in embryonic stages and that reduced Ptf1a dosage impairs exocrine development $(39,43)$, it is possible that Notch/Rbpj/Hes1-mediated Ptf1a repression prevents acinar cell differentiation in adult duct/centroacinar cells. If this is the case, a similar mechanism may operate in controlling the differentiation capacity of adult Sox $9^{+}$centroacinar cells as in embryonic stages. As mentioned, there exists mutual regulation among Sox9, Notch, and Hes1 during embryonic pancreatogenesis (Figure 2). In Sox9-CreER knock-in mice, reduced Sox 9 expression in adult centroacinar cells may result in reduced Notch-Hes 1 activity, resulting in continuous acinar cell differentiation through Ptfla acceleration. 
Along these lines, cell turnover in the adult organ would be rapid in a Sox9-IRES-CreER knock-in mouse, since its organ size is not apparently bigger than that of the wild-type animal. Indeed, the percentage of proliferating Sox $9^{+}$cells is reported to decrease in adult liver in BAC Sox9-CreER transgenic mice (44), but our preliminary results showed more $\mathrm{pHH} 3$-positive cells in the liver and pancreas in adult Sox9-IRES-CreER knock-in mice compared with the adult wildtype mice (Shinichi Hosokawa and Masashi Horiguchi, personal communication). Since the roles of Sox 9 in adult pancreas and liver are not well known, a dosage control of Sox9 in adult organs may be an open area for further investigation, not only in physiological organ homeostasis but also in regeneration and carcinogenesis.

\section{Duct cells in the regeneration of liver and pancreas}

As mentioned above, it is still unclear whether duct cells function as physiological progenitors in adult liver and pancreas. However, accumulating evidence supports the existence of precursors in the ducts that can respond to tissue injuries and participate in the restoration process, although the extent of their contribution differs depending on the type and degree of injury. For example, pancreatic Sox $9^{+}$duct cells do not differentiate into endocrine cells after pancreatic duct ligation, partial pancreatectomy, cerulein-induced pancreatitis, or in a streptozotocin-induced diabetes model $(22,69)$. After extensive $\beta$ cell ablation using diphtheria toxin receptor-expressing transgenic mice, transdifferentiation of $\alpha$ cells into $\beta$ cells was observed (70), but not differentiation of Sox $9^{+}$duct cells into $\beta$ cells (69). In contrast, extensive diphtheria toxin-induced depletion of both acinar and endocrine cells combined with $P d x 1$-Cre as a lineage-tracing system showed that the remaining cells in the ductal component contributed to the regeneration of both endocrine and acinar cells (10).

In Sox9-IRES-CreER knock-in mice, proliferation of the Sox9 $9^{+}$bile duct cells and subsequent hepatocyte differentiation is accelerated by injuries including acute carbon tetrachloride $\left(\mathrm{CCl}_{4}\right)$, bile duct ligation, and methionine- and choline-deficient diet supplemented with $0.15 \%$ ethionine. Other injury models such as $70 \%$ partial hepatectomy, 3,5-diethoxycarbonyl-1,4-dihydrocollidine-supplemented (DDC-supplemented) diet, and acetaminophen treatment result in moderate or low contribution of Sox9 $9^{+}$duct cells to the regenerating tissue (22). In the DDC model, accelerated hepatocyte differentiation of Sox $9^{+}$cells was also observed in the BAC Sox9-CreER transgenic mice (24). Of note, Malato et al. detected lineage-negative hepatocytes, although small in number, emerging after some hepatic injuries in AAV-Ttr-Cre mice (61). They showed that non-hepatocyte cells contributed to organ repair after chronic $\mathrm{CCl}_{4}$ exposure, 2/3 partial hepatectomy, bile duct ligation of the left lobe, and DDC diet, and they speculated that these newly generated cells are of duct cell origin (61). Overall, these findings are in accordance with the results obtained by Sox9-IRES-CreER mice with the exception in $\mathrm{CCl}_{4}$ models; Malato et al. found contribution of non-hepatocyte cells after chronic $\mathrm{CCl}_{4}$ exposure but not by the acute $\mathrm{CCl}_{4}$ model (61), whereas Sox9 $9^{+}$bile duct cells function as the source of hepatocyte regeneration by the acute $\mathrm{CCl}_{4}$ model in Sox9-IRES-CreER knock-in mice (22).

\section{Future perspectives}

Many researchers in the field have suggested that duct cells are the best candidates for the origin of human pancreas ductal adenocarcinoma (PDAC) and cholangiocarcinoma (CCC). However, in mouse models, accumulating evidence suggests that metaplasia of differentiated acinar cells and hepatocytes to a ductal phenotype is the key event in the initiation of PDAC and CCC, respectively (71-78). In this process, Notch appears to play a fundamental role; inactivation or activation of Notch signaling in acinar cells and hepatocytes does not cause any lesions, but the combination of Notch activation and carcinogenic signals (oncogenic Kras induction in acinar cells and thioacetamide administration or AKT overexpression in hepatocytes) results in conversion to a duct cell type (76-78). Interestingly, Sox9 is overexpressed in human hepatocellular carcinoma, and its expression is associated with tumor progression and poor prognosis (79). More recently, ectopic expression of Sox 9 and Hnf6, another pancreatic duct marker, has been reported in human metaplastic acinar cells associated with pancreatitis and pancreatic adenocarcinoma (80). Gain- and loss-of-function experiments using culture and mice suggest that Hnf6, and to a lesser extent Sox9, are required for the acinar-to-ductal metaplasia (80). The significance of Sox9 in other tumors and that in the experimental models is an area that warrants further investigation.

In summary, Sox9 plays a pivotal role in the programming of embryonic liver and pancreas progenitors. It is still unclear whether Sox $9^{+}$duct cells function as physiological progenitors in adult organ homeostasis, but these cells appear to be activated during the tissue regeneration process. Uncovering more mechanistic detail on the maintenance of the precursor status of embryonic Sox $9^{+}$cells and/or manipulation of Sox 9 expression in adult cells may provide a basis for exploring new therapies for human diseases of the liver and pancreas.

\section{Acknowledgments}

I thank Maureen Gannon (Vanderbilt University) for critical reading of the manuscript.

Address correspondence to: Yoshiya Kawaguchi, Department of Clinical Application, Center for iPS cell Research and Application (CiRA), Kyoto University, Development 2012, 53 Kawahara-cho, Shogoin, Sakyo-ku, Kyoto, 606-8507, Japan. Phone: 81.75.366.7049; Fax: 81.75.366.7050; E-mail: yoshiyak@cira.kyoto-u.ac.jp.
1. Barker N, et al. Crypt stem cells as the cells-of-origin of intestinal cancer. Nature. 2009;457(7229):608-611.

2. Chen J, et al. A restricted cell population propagates glioblastoma growth after chemotherapy. Nature. 2012;488(7412):522-526.

3. Driessens G, Beck B, Caauwe A, Simons BD, Blanpain C. Defining the mode of tumour growth by clonal analysis. Nature. 2012;488(7412):527-530.

4. Schepers AG, et al. Lineage tracing reveals Lgr5+ stem cell activity in mouse intestinal adenomas. Science. 2012;337(6095):730-735.

5. Baker M. Cancer stem cells tracked. Nature. 2012; 488(7409):13-14.

6. Jensen JN, Cameron E, Garay MV, Starkey TW, Gianani R, Jensen J. Recapitulation of elements of embryonic development in adult mouse pancreatic regeneration. Gastroenterology. 2005;128(3):728-741. 7. Thiery JP, Acloque H, Huang RY, Nieto MA. Epithelial-mesenchymal transitions in development and disease. Cell. 2009;139(5):871-890.

8. Acloque H, Adams MS, Fishwick K, Bronner-Fraser M, Nieto MA. Epithelial-mesenchymal transitions: the importance of changing cell state in development and disease. J Clin Invest. 2009;119(6):1438-1449.

9. Li WC, et al. Activation of pancreatic-duct-derived progenitor cells during pancreas regeneration in adult rats. J Cell Sci. 2010;123(pt 16):2792-2802.

10. Criscimanna A, et al. Duct cells contribute to regeneration of endocrine and acinar cells following pancreatic damage in adult mice. Gastroenterol- ogy. 2011;141(4):1451-1462

11. Wagner T, et al. Autosomal sex reversal and campomelic dysplasia are caused by mutations in and around the SRY-related gene SOX9. Cell. 1994;79(6):1111-1120.

12. Lefebvre V, Dumitriu B, Penzo-Mendez A, Han Y, Pallavi B. Control of cell fate and differentiation by Sry-related high-mobility-group box (Sox) transcription factors. Int J Biochem Cell Biol. 2007;39(12):2195-2214.

13. Akiyama H. Control of chondrogenesis by the transcription factor Sox9. Mod Rheumatol. 2008;18(3):213-219.

14. Lincoln J, Kist R, Scherer G, Yutzey KE. Sox9 is required for precursor cell expansion and extracellular 
matrix organization during mouse heart valve development. Dev Biol. 2007;305(1):120-132.

15. Montero JA, et al. Expression of Sox8, Sox 9 and Sox 10 in the developing valves and autonomic nerves of the embryonic heart. Mech Dev. 2002;118(1-2):199-202.

16. Seymour PA, et al. SOX9 is required for maintenance of the pancreatic progenitor cell pool. Proc Natl Acad Sci U S A. 2007;104(6):1865-1870.

17. Antoniou $A$, et al. Intrahepatic bile ducts develop according to a new mode of tubulogenesis regulated by the transcription factor SOX9. Gastroenter ology. 2009;136(7):2325-2333

18. Vidal VP, et al. Sox 9 is essential for outer root sheath differentiation and the formation of the hair stem cell compartment. Curr Biol. 2005;15(15):1340-1351.

19. Poche RA, Furuta Y, Chaboissier MC, Schedl A, Behringer RR. Sox 9 is expressed in mouse multipotent retinal progenitor cells and functions in Muller glial cell development. J Comp Neurol. 2008;510(3):237-250.

20. Stolt CC, Lommes P, Sock E, Chaboissier MC, Schedl A, Wegner M. The Sox9 transcription factor determines glial fate choice in the developing spinal cord. Genes Dev. 2003;17(13):1677-1689.

21. Perl AK, Kist R, Shan Z, Scherer G, Whitsett JA. Normal lung development and function after Sox 9 inactivation in the respiratory epithelium. Genesis. 2005;41(1):23-32.

22. Furuyama K, et al. Continuous cell supply from a Sox9-expressing progenitor zone in adult liver, exocrine pancreas and intestine. Nat Genet. 2011;43(1):34-41.

23. Kopp JL, et al. Sox9+ ductal cells are multipotent progenitors throughout development but do not produce new endocrine cells in the normal or injured adult pancreas. Development. 2011;138(4):653-665.

24 . Dorrell C, et al. Prospective isolation of a bipotential clonogenic liver progenitor cell in adult mice. Genes Dev. 2011;25(11):1193-1203.

25. Kawaguchi Y, Takaori K, Uemoto S. Genetic lineage tracing, a powerful tool to investigate the embryonic organogenesis and adult organ maintenance of the pancreas. J Hepatobiliary Pancreat Sci. 2011;18(1):1-5.

26. Soriano P. Generalized lacZ expression with the ROSA26 Cre reporter strain. Nat Genet 1999;21(1):70-71.

27. Seymour PA, Freude KK, Dubois CL, Shih HP, Patel NA, Sander M. A dosage-dependent requirement for Sox9 in pancreatic endocrine cell formation. Dev Biol. 2008;323(1):19-30.

28. Piper K, Ball SG, Keeling JW, Mansoor S, Wilson DI, Hanley NA. Novel SOX9 expression during human pancreas development correlates to abnormalities in Campomelic dysplasia. Mech Dev. 2002;116(1-2):223-226.

29. Foster JW, et al. Campomelic dysplasia and autosomal sex reversal caused by mutations in an SRY-related gene. Nature. 1994;372(6506):525-530.

30. Bi W, et al. Haploinsufficiency of Sox9 results in defective cartilage primordia and premature skeletal mineralization. Proc Natl Acad Sci U S A. 2001;98(12):6698-6703.

31. Lynn FC, Smith SB, Wilson ME, Yang KY, Nekrep $\mathrm{N}$, German MS. Sox 9 coordinates a transcriptional network in pancreatic progenitor cells. Proc Natl Acad Sci U S A. 2007;104(25):10500-10505.

32. Shih HP, et al. A Notch-dependent molecular circuitry initiates pancreatic endocrine and ductal cell differentiation. Development. 2012;139(14):2488-2499.

33. Seymour PA, et al. A Sox9/Fgf feed-forward loop maintains pancreatic organ identity. Development 2012;139(18):3363-3372.

34. Delous M, et al. Sox9b is a key regulator of pancreaticobiliary ductal system development. PLoS Genet. 2012;8(6):e1002754.

35. Manfroid I, et al. Zebrafish sox $9 b$ is crucial for hepatopancreatic duct development and pancreatic endocrine cell regeneration. Dev Biol. 2012;366(2):268-278

36. Lee JC, et al. Regulation of the pancreatic pro-endocrine gene neurogenin3. Diabetes. 2001;50(5):928-936. 37. Apelqvist A, et al. Notch signalling controls pancreatic cell differentiation. Nature. 1999; 400(6747):877-881

38. Kawaguchi Y, Cooper B, Gannon M, Ray M, MacDonald RJ, Wright CV. The role of the transcriptional regulator Ptf1a in converting intestinal to pancreatic progenitors. Nat Genet. 2002;32(1):128-134.

39. Fukuda A, et al. Reduction of Ptf1a gene dosage causes pancreatic hypoplasia and diabetes in mice. Diabetes. 2008;57(9):2421-2431.

40. Krapp A, et al. The bHLH protein PTF1-p48 is essential for the formation of the exocrine and the correct spatial organization of the endocrine pancreas. Genes Dev. 1998;12(23):3752-3763.

41. Masui T, Swift GH, Hale MA, Meredith DM, Johnson JE, Macdonald RJ. Transcriptional autoregulation controls pancreatic Ptf1a expression during development and adulthood. Mol Cell Biol. 2008;28(17):5458-5468

42. Masui T, et al. Replacement of Rbpj with Rbpjl in the PTF1 complex controls the final maturation of pancreatic acinar cells. Gastroenterology. 2010;139(1):270-280

43. Fukuda A, et al. Ectopic pancreas formation in Hes 1 -knockout mice reveals plasticity of endodermal progenitors of the gut, bile duct, and pancreas. J Clin Invest. 2006;116(6):1484-1493.

44. Carpentier R, et al. Embryonic ductal plate cells give rise to cholangiocytes, periportal hepatocytes, and adult liver progenitor cells. Gastroenterology. 2011;141(4):1432-1438

45. Kodama Y, Hijikata M, Kageyama R, Shimotohno $\mathrm{K}$, Chiba T. The role of notch signaling in the development of intrahepatic bile ducts. Gastroenterology. 2004;127(6):1775-1786

46. Oda T, et al. Mutations in the human Jagged 1 gene are responsible for Alagille syndrome. Nat Genet. 1997;16(3):235-242.

47. Geisler F, et al. Liver-specific inactivation of Notch2, but not Notch1, compromises intrahepatic bile duct development in mice. Hepatology. 2008;48(2):607-616.

48. Zong Y, et al. Notch signaling controls liver development by regulating biliary differentiation. Development. 2009;136(10):1727-1739.

49. Mascre G, et al. Distinct contribution of stem and progenitor cells to epidermal maintenance. Nature. 2012;489(7415):257-262.

50 . Barker $\mathrm{N}$, et al. Identification of stem cells in small intestine and colon by marker gene Lgr5. Nature. 2007;449(7165):1003-1007.

51. Alison MR, Marongiu F, Laconi E. Transplanted hepatocytes: wiped out or washed out? J Hepatol. 2012;56(4):996-997.

52. Zajicek G, Oren R, Weinreb M. The streaming liver. Liver. 1985;5(6):293-300.

53. Arber N, Zajicek G, Ariel I. The streaming liver. II. Hepatocyte life history. Liver. 1988;8(2):80-87.

54. Zajicek G, Ariel I, Arber N. The streaming liver. III. Littoral cells accompany the streaming hepatocyte. Liver. 1988;8(4):213-218.

55. Schwartz-Arad D, Zajicek G, Bartfeld E. The streaming liver IV: DNA content of the hepatocyte increases with its age. Liver. 1989;9(2):93-99.

56. Zajicek G, Schwartz-Arad D, Bartfeld E. The streaming liver. V: Time and age-dependent changes of hepatocyte DNA content, following partial hepatectomy. Liver. 1989;9(3):164-171.

57. Arber N, Zajicek G. Streaming liver. VI: Streaming intra-hepatic bile ducts. Liver. 1990;10(4):205-208.

58. Zajicek G, Schwartz-Arad D. Streaming liver. VII: DNA turnover in acinus zone-3. Liver. 1990;10(3):137-140.
59. Bralet MP, Branchereau S, Brechot C, Ferry N. Cell lineage study in the liver using retroviral mediated gene transfer. Evidence against the streaming of hepatocytes in normal liver. Am J Pathol. 1994;144(5):896-905

60. Fellous TG, et al. Locating the stem cell niche and tracing hepatocyte lineages in human liver. Hepatology. 2009;49(5):1655-1663.

61. Malato Y, et al. Fate tracing of mature hepatocytes in mouse liver homeostasis and regeneration. J Clin Invest. 2011;121(12):4850-4860.

62. Iverson SV, Comstock KM, Kundert JA, Schmidt EE. Contributions of new hepatocyte lineages to liver growth, maintenance, and regeneration in mice. Hepatology. 2011;54(2):655-663.

63. Solar M, et al. Pancreatic exocrine duct cells give rise to insulin-producing beta cells during embryogenesis but not after birth. Dev Cell. 2009; 17(6):849-860.

64. Dor Y, Brown J, Martinez OI, Melton DA. Adult pancreatic beta-cells are formed by self-duplication rather than stem-cell differentiation. Nature. 2004;429(6987):41-46.

65. Desai BM, et al. Preexisting pancreatic acinar cells contribute to acinar cell, but not islet beta cell, regeneration. J Clin Invest. 2007;117(4):971-977.

66. Teta M, Rankin MM, Long SY, Stein GM, Kushner JA. Growth and regeneration of adult beta cells does not involve specialized progenitors. Dev Cell. 2007;12(5):817-826.

67. Kopinke D, Brailsford M, Shea JE, Leavitt R, Scaife CL, Murtaugh LC. Lineage tracing reveals the dynamic contribution of Hes1+ cells to the developing and adult pancreas. Development. 2011;138(3):431-441.

68. Kopinke D, Brailsford M, Pan FC, Magnuson MA, Wright CV, Murtaugh LC. Ongoing Notch signaling maintains phenotypic fidelity in the adult exocrine pancreas. Dev Biol. 2012;362(1):57-64

69. Kopp JL, Dubois CL, Hao E, Thorel F, Herrera PL, Sander M. Progenitor cell domains in the developing and adult pancreas. Cell Cycle. 2011;10(12):1921-1927.

70. Thorel F, et al. Conversion of adult pancreatic alpha-cells to beta-cells after extreme beta-cell loss. Nature. 2010;464(7292):1149-1154.

71. Grippo PJ, Nowlin PS, Demeure MJ, Longnecker DS, Sandgren EP. Preinvasive pancreatic neoplasia of ductal phenotype induced by acinar cell targeting of mutant Kras in transgenic mice. Cancer Res. 2003;63(9):2016-2019.

72. Shi G, et al. Loss of the acinar-restricted transcription factor Mist 1 accelerates Kras-induced pancreatic intraepithelial neoplasia. Gastroenterology. 2009;136(4):1368-1378.

73. Hill R, et al. PTEN loss accelerates KrasG12D-induced pancreatic cancer development. Cancer Res. 2010;70(18):7114-7124

74. Morris JP 4th, Cano DA, Sekine S, Wang SC, Hebrok $\mathrm{M}$. Beta-catenin blocks Kras-dependent reprogramming of acini into pancreatic cancer precursor lesions in mice. J Clin Invest. 2010;120(2):508-520.

75. Corcoran RB, et al. STAT3 plays a critical role in KRAS-induced pancreatic tumorigenesis. Cancer Res. 2011;71(14):5020-5029.

76. De La O JP, et al. Notch and Kras reprogram pancreatic acinar cells to ductal intraepithelial neoplasia. Proc Natl Acad Sci U S A. 2008;105(48):18907-18912.

77. Sekiya S, Suzuki A. Intrahepatic cholangiocarcinoma can arise from Notch-mediated conversion of hepatocytes. J Clin Invest. 2012;122(11):3914-3918.

78. Fan B, et al. Cholangiocarcinomas can originate from hepatocytes in mice. J Clin Invest. 2012;122(8):2911-2915

79. Guo X, et al. Expression features of SOX9 associate with tumor progression and poor prognosis of hepatocellular carcinoma. Diagn Pathol. 2012;7:44.

80. Prevot PP, et al. Role of the ductal transcription factors HNF6 and Sox9 in pancreatic acinar-to-ductal metaplasia. Gut. 2012;61(12):1723-1732. 